\title{
IUFOST2006/871 The dimensions of food microstructure
}

\author{
J.M. Aguilera \\ Dep. Chemical Eng. and Bioprocess, Universidad Católica de Chile, Vicuña Mackenna 4860, PO Box 306 \\ Lund, Chile \\ jmaguile@ing.puc.cl
}

Quality and health are two main drivers of the modern food industry and food microstructure influences both. The preservation of the natural microstructure of cereals, tubers, fruits, vegetables and muscle foods is of primary importance and the target of postharvest and postmortem technologies. These foods are hierarchically structured from the molecular to the macro-level and most of their desirable properties depend on how food microstructure is preserved during the transit from producers to consumers. For the processed food industry that transforms and creates food structures the range of relevant dimensions spans from nanometer scale (e.g., flavors, surfactants) to whole products (and equipment). Time scales may vary between the microseconds, for example, in relaxation phenomena in glassy domains, to months in the case of stored products. As health and wellbeing derived from food consumption become increasingly relevant for the food industry, the brain-gut axis is now object of much attention. Structure breakdown in the mouth and gut is central to the release of flavors and nutrients as well as in gastrointestinal transit and nutrient bioavailability. This presentation will review the tools and techniques presently used to resolve the role of food microstructure at the required length and time scales in the producer-consumer and brain-gut axes. 